\title{
Optimal Multi-UAV Convoy Protection
}

\author{
X. C. Ding, A. Rahmani and M. Egerstedt
}

\begin{abstract}
In this paper, we study time-optimal trajectories for Unmanned Aerial Vehicles (UAVs) to provide convoy protection to a group of stationary ground vehicles. The UAVs are modelled as Dubins vehicles flying at a constant altitude. Due to kinematic constraints of the UAVs, it is not possible for a single UAV to provide convoy protection indefinitely. In this paper, we derive time-optimal paths for a single UAV to provide continuous ground convoy protection for the longest possible time. Furthermore, this paper provides optimal trajectories for multiple UAVs to achieve uninterrupted convoy protection. The minimum number of UAVs required to achieve this task is determined.
\end{abstract}

\section{INTRODUCTION}

In a number of future civilian and military applications, it is expected that heterogeneous, unmanned vehicles have to be coordinated despite their highly varying dynamics and sensing capabilities. In particular, UGVs (Unmanned Ground Vehicles) are expected to solve such tasks as surveillance, clearing of hostile terrains, transportation in convoys, and the establishment of logistic support chains. At the same time, the UGVs will need to interact with more dynamically capable UAVs (Unmanned Aerial Vehicles) to give them access to large-scale area surveillance, convoy protection, and advanced scouting capabilities ([7], [15]). There arises naturally the problem of control and coordination of UAVs in order to provide ground convoy protection to the UGVs in an optimal manner, while satisfying the kinematic constraints due to nonholonomic dynamics of the UAVs. The goal of this paper is to provide the optimal control strategy for a single UAV, as well as optimal path planning for multiple UAVs in order to provide successful convoy protection.

In this paper we address the problem when the ground vehicles are stationary, and the convoy protection is defined as providing coverage for some given ground vehicles. The UAVs are modelled as Dubins vehicles [4] flying at a constant altitude. Due to kinematic constraints of the UAVs and limited ranges of sensors on-board the UAVs, it may be impossible to provide coverage to the ground vehicles with a single UAV. In this case, the problem of interest becomes that of providing an optimal path for a single UAV so that it can monitor the ground vehicle for the longest amount of time, and coordinating multiple UAVs so that the ground vehicles are visible to at least one UAV at any given time. Figure 1 illustrates 2 UAVs flying a circular path of radius $R$ while providing coverage to a group of UGVs.

X.C. Ding, A. Rahmani, and M. Egerstedt are with the School of Electrical and Computer Engineering, Georgia Institute of Technology, Atlanta, GA 30308; Emails: \{ding,arahmani,magnus\}@ece.gatech.edu. The research of these authors has been supported by a grant from Rockwell Collins Advanced Technology Center.

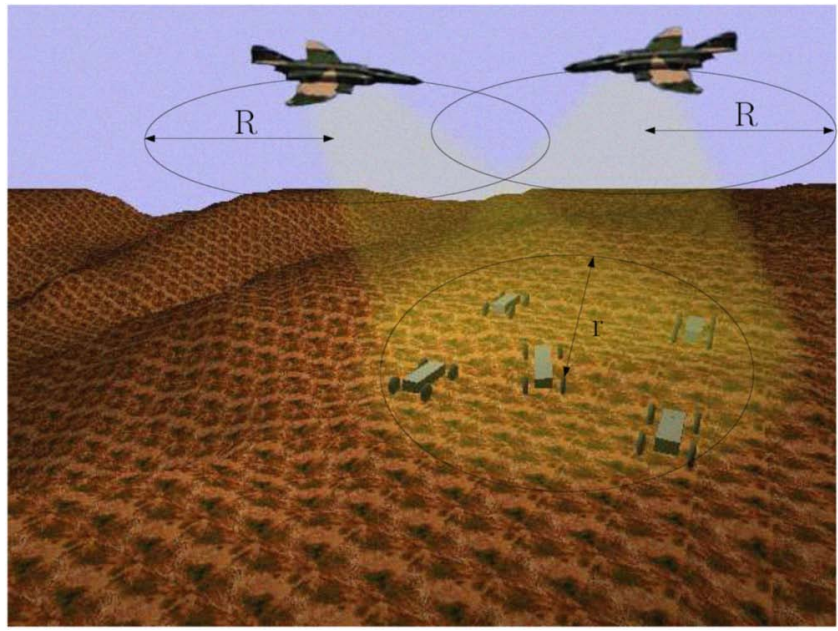

Fig. 1. Illustration of 2 UAVs providing convoy protection to UGVs.

A Dubins vehicle is a planar vehicle with bounded turning radius and constant speed that always moves forward. L.E. Dubins was the first to give a characterization of time-optimal trajectories for such a vehicle using geometric methods [4]. Shortest-path problems for Dubins vehicles have been since studied extensively (e.g. [5], [12]). A Dubins vehicle that can move backwards was studied by Reeds and Shepp [11], and the shortest-path problem for a Reeds-Shepp vehicle was further studied and classified by Souères and Laumond [13]. Walsh et al. found optimal paths for an airplane on $S E(2)$ [16]. Dubins vehicle has been used as a simplified model to describe planar motion of UAVs [12], [2]. Chitsaz et al. extend the Dubins' model from $S E(2)$ to $S E(2) \times \mathbb{R}$ to account for altitude changes and gave a characterization of the timeoptimal trajectories for this model based on the final altitude [2].

An important approach to solve problems involving Dubins vehicle is to use motion primitives to produce optimal trajectories (e.g. [1], [6], [8]). In the case of minimum time point-topoint transfer problem for Dubins car, Pontryagin minimum principle provides a set of necessary optimality conditions which state that the optimal solutions are curves consisting only three motion primitives: line-segment and circular arcs turning maximumly to the left and to the right [10], [14]. We will use the same idea of motion primitives to construct optimal trajectories, but it will be seen later that line-segments are not part of optimal trajectories for this problem.

The technical report [3] describes the content of this paper in more details and it contains all the proofs.

The rest of the paper is organized as follows. Section II 
formulates the problem. In Section III the optimal solution for controlling a single UAV for maximum-time convoy protection is proposed. Section IV proposes optimal paths for multiple UAVs to provide successful convoy protection for all time. Section V concludes the paper.

\section{PROBlem Formulation}

In this paper, the UAVs in question are modelled as Dubins vehicles flying at a constant altitude and they follow paths of bounded curvature. The minimum turning radius of the UAV is assumed to be $R$. Without loss of generality, we assume the vehicles to be of unit speed. ${ }^{1}$ The kinematic equations of the Dubins vehicle are written as:

$$
\left\{\begin{array}{l}
\dot{x}=\cos (\theta) \\
\dot{y}=\sin (\theta) \\
\dot{\theta}=\omega
\end{array}\right.
$$

where $x$ and $y$ are the position of the UAV in the $x-y$ plane on the altitude the UAV is flying, and $\omega$ is the angular velocity of the vehicle. The angular velocity is bounded by the inverse of the minimum turn radius $R$ of the vehicle, i.e., $\omega \in\left[-\frac{1}{R}, \frac{1}{R}\right]$. Hence, the paths of the UAVs are curves with curvature bounded by $\left[0, \frac{1}{R}\right]$. The state of the system is defined as $q(t)=[x(t), y(t), \theta(t)]^{T}$.

We assume that the UAVs have cameras or other sensors rigidly mounted on-board the vehicle, and the camera monitors a disk of radius $r$ on the ground. The ground vehicles are assumed to be stationary, and the centroid of the vehicles are defined to be the point which must be visible to the UAVs. Convoy protection is considered to be provided when the centroid of the UGVs is visible to the UAV and inside the aforementioned circle defined by the camera. Successful convoy coverage is achieved if the centroid of the ground vehicles is visible to at least one UAV at any time.

In many cases, cameras or sensors on-board the vehicle have narrower field of vision than the UAV's turning radius, especially for cameras and sensors that carry out a specific objective, for example executing computer vision algorithms. If $R>r$, then a single UAV is not capable of providing convoy protection to the ground vehicles indefinitely, and a control strategy is needed to optimize the time in which convoy protection is achieved. This is assumed to be the case throughout the paper. Note that if $R \leq r$, then the convoy protection problem is solved by using a single UAV flying on a circular path of radius $R$ with the center being the ground vehicles.

This paper considers the problem of finding optimal trajectories of a single UAV to maximize the time in which the ground vehicles are visible, and optimal paths for multiple UAVs so that the UGVs are visible to at least one UAV at any time. There is an equivalent reformulation of this problem. Fix a circle of radius $r$ centered at the centroid of UGVs. This circle is denoted as the convoy circle. The ground vehicles are visible to a UAV if the distance between

\footnotetext{
${ }^{1}$ The unit speed assumption is justified since the results presented in this paper describe paths, and they are invariant under different forward speed.
}

the UAV and the centroid in the $x-y$ plane is less or equal to $r$. Thus, the problem can be reformulated as finding time-optimal trajectories of a UAV so that the time the UAV remains inside the convoy circle is maximized.

\section{OPTIMAL PATHS FOR A Single UAV}

In this section we consider the problem of finding the optimal paths for a single UAV to maximize the time it stays in the convoy circle. We will determine both the time-optimal path for a single UAV starting at a fixed initial condition and optimal paths if the UAV is allowed to pick the position and heading when entering the convoy circle.

Fix the origin of the $x-y$ plane at the centroid of the UGVs. This problem can be considered as an optimal control problem with state constraint $x^{2}+y^{2}-r^{2} \leq 0$ and input constraint $|w| \leq \frac{1}{R}$. Furthermore, it can be assumed that the UAV starts at a point on the state constraint boundary (convoy circle), since we are interested in complete trajectories of UAVs inside the convoy circle.

The problem at hand is the following (maximum time) optimal control problem with the cost function defined as

$$
\min _{\omega(t)} J=\int_{0}^{T}-1 d t
$$

subject to the dynamics of (1), and the input and state constraints

$$
\begin{array}{r}
-\frac{1}{R}<\omega(t)<\frac{1}{R}, \\
x(t)^{2}+y(t)^{2}-r^{2} \leq 0,
\end{array}
$$

with given $r<R$ and initial condition $q(0)$ satisfying the state constraint $x(0)^{2}+y(0)^{2}-r^{2}=0$. We henceforth denote this problem as $\Pi_{q(0)}$.

We exclude initial conditions that generate no path entering the convoy circle. This occurs when the initial heading $\theta(0)$ points away from the convoy circle. The set of initial conditions $\Lambda$ that are considered for the optimization problem can be defined as:

$$
\begin{aligned}
\Lambda= & \left\{q=[x, y, \theta]^{T}: x^{2}+y^{2}=r^{2},\right. \text { and } \\
& \left.-\frac{\pi}{2}<\theta-\operatorname{atan} 2(y, x)<\frac{\pi}{2}\right\}
\end{aligned}
$$

The set $\Lambda$ is denoted as the feasible entry set. For simplicity of notation, we assume that all angles are taken modulus $2 \pi$.

State inequality constraint optimal control problems are usually hard or impossible to be solved explicitly. In this problem, the special structure of the problem provides that the constrained arc of the solution is at only two points, initial condition and when the UAV is exiting the circle. Due to this special structure, the state constraint for this problem is handled by an auxiliary state. Define $\xi\left(x^{2}+y^{2}-r^{2}\right)$ as an inverted Heaviside function:

$$
\xi\left(x^{2}+y^{2}-r^{2}\right)=\left\{\begin{array}{l}
0: x^{2}+y^{2}-r^{2} \leq 0 \\
1: \text { otherwise. }
\end{array}\right.
$$

Define a new state $\tau(t)$ as:

$$
\dot{\tau}(t)=\left(x^{2}+y^{2}-r^{2}\right)^{2} \xi\left(x^{2}+y^{2}-r^{2}\right),
$$


then the state can be augmented as $\bar{q}(t)=[q(t), \tau(t)]^{T}$. Let us require that $\tau(0)=0$ and $\tau(T)=0$. This enforces the constraint since being outside of the constraint produces a positive derivative of $\tau(t)$ and thus the terminal condition is violated. When there is no ambiguity, we assume that the state constraint is satisfied and we still call $q(t)$ the state trajectory.

To help the analysis, it is useful to impose an extra terminal manifold constraint. Since the optimal solution always involves the terminal state (henceforth denoted as the exit state) being on the boundary of the state constraint set (exiting the circle), the terminal constraint of being on the convoy circle when exiting is enforced. For simplicity of notation, denote $q_{T}:=q(T)$ and $\left[x_{T}, y_{T}, \theta_{T}\right]:=[x(T), y(T), \theta(T)]$. Using this notation, the terminal manifold can be defined as a set of states that satisfies:

$$
M\left(q_{T}\right)=x_{T}^{2}+y_{T}^{2}-r^{2}=0 .
$$

The Hamiltonian for this optimal control problem can be written as:

$$
\begin{aligned}
\mathcal{H}= & -1+\lambda_{1} \cos \theta+\lambda_{2} \sin \theta+\lambda_{3} \omega+ \\
& \lambda_{4}\left(x^{2}+y^{2}-r^{2}\right)^{2} \xi\left(x^{2}+y^{2}-r^{2}\right),
\end{aligned}
$$

where $\lambda=\left[\lambda_{1}, \cdots, \lambda_{4}\right]^{T}$ are the trajectories of costates. The necessary optimality condition from the Pontryagin's minimum principle states that

$$
\begin{aligned}
\mathcal{H}\left(\bar{q}^{*}(t), \lambda^{*}(t), \omega^{*}(t), t\right) & \leq \mathcal{H}\left(\bar{q}^{*}(t), \lambda^{*}(t), \omega(t), t\right), \\
& \forall \omega \in\left[-\frac{1}{R}, \frac{1}{R}\right], t \in[0, T],
\end{aligned}
$$

where the costates are obtained as:

$$
\begin{aligned}
& \dot{\lambda}_{1}=-\frac{\partial \mathcal{H}}{\partial x}=-2 x \lambda_{4}\left(x^{2}+y^{2}-r^{2}\right) \xi\left(x^{2}+y^{2}-r^{2}\right) \\
& \dot{\lambda}_{2}=-\frac{\partial \mathcal{H}}{\partial y}=-2 y \lambda_{4}\left(x^{2}+y^{2}-r^{2}\right) \xi\left(x^{2}+y^{2}-r^{2}\right) \\
& \dot{\lambda}_{3}=-\frac{\partial \mathcal{H}}{\partial \theta}=\lambda_{1} \sin \theta-\lambda_{2} \cos \theta \\
& \dot{\lambda}_{4}=-\frac{\partial \mathcal{H}}{\partial \tau}=0
\end{aligned}
$$

Using the necessary optimality condition, and substituting the Hamiltonian from (9), one can see that the optimal controller is a function of the costate $\lambda_{3}(t)$ as:

$$
\omega^{*}(t)= \begin{cases}-\frac{1}{R} & : \lambda_{3}^{*}(t)>0 \\ \frac{1}{R} & : \lambda_{3}^{*}(t)<0\end{cases}
$$

Thus it can be seen that when $\lambda_{3}^{*}(t)>0$, the optimal control is maximum turning right, and when $\lambda_{3}^{*}(t)<0$, the optimal control is maximum turning left. Hence, the optimal control trajectory is in the form of bang-bang control. It should be noted that when $\lambda_{3}^{*}(t)=0$ for a finite time interval, then any control $w(t)$ satisfies (10) and this case is referred to as a singular condition (see [9]). For a singular condition to occur, it is necessary that there exist a time $t$ such that $\lambda(t)=0$ and $\dot{\lambda}(t)=0$. For Dubins vehicle problems, this results in line segments and we elaborate later in this section when this scenerio arises.
Definition 3.1: For a state trajectory $q(t), t \in[0, T]$ satisfying the state constraint (4), if the costate trajectory and corresponding input satisfies the control strategy (11), then $q(t)$ is referred to as a Candidate Optimal Trajectory (COT).

Pontryagin's minimum principle states that being a COT is a necessary condition for being the optimal solution.

When the state constraint is satisfied, i.e. the UAV is inside the convoy circle, the first three costate equations are independent from the augmented state and costate equations, and they can be rewritten as

$$
\begin{aligned}
& \dot{\lambda}_{1}=0 \\
& \dot{\lambda}_{2}=0 \\
& \dot{\lambda}_{3}=\lambda_{1} \sin (\theta)-\lambda_{2} \cos (\theta)
\end{aligned}
$$

with the terminal condition:

$$
\begin{aligned}
\lambda_{1}(T) & =\frac{\partial M}{\partial x}\left(x_{T}\right)=2 x_{T} \alpha \\
\lambda_{2}(T) & =\frac{\partial M}{\partial y}\left(y_{T}\right)=2 y_{T} \alpha \\
\lambda_{3}(T) & =\frac{\partial M}{\partial \theta}\left(\theta_{T}\right)=0,
\end{aligned}
$$

where $\alpha$ is a constant Lagrange multiplier for the terminal manifold.

Furthermore, being a minimum-time optimal control problem the transversality condition gives that

$$
\begin{aligned}
\left.\mathcal{H}\right|_{t=T}= & -1+\lambda_{1}(T) \cos \left(\theta_{T}\right)+\lambda_{2}(T) \sin \left(\theta_{T}\right) \\
& +\lambda_{3}(T) \omega(T)=0
\end{aligned}
$$

which implies that:

$$
\alpha=\frac{1}{2\left(x_{T} \cos \left(\theta_{T}\right)+y_{T} \sin \left(\theta_{T}\right)\right)} .
$$

Thus,

$$
\begin{gathered}
\lambda_{1}=\frac{x_{T}}{x_{T} \cos \left(\theta_{T}\right)+y_{T} \sin \left(\theta_{T}\right)}, \forall t \in[0, T] \\
\lambda_{2}=\frac{y_{T}}{x_{T} \cos \left(\theta_{T}\right)+y_{T} \sin \left(\theta_{T}\right)}, \forall t \in[0, T] .
\end{gathered}
$$

Therefore, $\lambda_{3}$ costate equation can be rewritten as:

$$
\dot{\lambda}_{3}=\frac{x_{T} \sin (\theta)-y_{T} \cos (\theta)}{x_{T} \cos \left(\theta_{T}\right)+y_{T} \sin \left(\theta_{T}\right)},
$$

with the initial condition $\lambda_{3}(T)=0$.

Define the angle $\psi_{T}=\operatorname{atan} 2\left(y_{T}, x_{T}\right)$. Note that since $q_{T}$ is an exit point, then by definition $-\pi / 2<\psi_{T}-\theta_{T}<\pi / 2$. If not, then $q_{T}$ is an entry point instead of an exit point. The case where $\left|\psi_{T}-\theta_{T}\right|=\pi / 2$ is excluded from consideration, since in this case $q_{T}$ is both an entry point and exit point, and it does not belong to the feasible entry set $\Lambda$.

Assuming a trajectory $q(t)$ is a COT and given the terminal state $q_{T}$, we will show that $\lambda(t)$ satisfying the necessary optimality conditions can be uniquely determined. First note 
that (15) can be simplified as quotient of two inner-products:

$$
\begin{gathered}
\dot{\lambda}_{3}(t)=\frac{\left\langle\left[\begin{array}{l}
x_{T} \\
y_{T}
\end{array}\right],\left[\begin{array}{c}
\sin (\theta(t)) \\
-\cos (\theta(t))
\end{array}\right]\right\rangle}{\left\langle\left[\begin{array}{l}
x_{T} \\
y_{T}
\end{array}\right],\left[\begin{array}{c}
\cos \left(\theta_{T}\right) \\
\sin \left(\theta_{T}\right)
\end{array}\right]\right\rangle} \\
=\frac{\sin \left(\theta(t)-\psi_{T}\right)}{\cos \left(\theta_{T}-\psi_{T}\right)}
\end{gathered}
$$

If $q(t)$ is a COT then $\omega(t)$ satisfies the optimal control strategy (12). In addition if $\lambda_{3}(t) \neq 0$ and has the same sign for a time interval $[s, T)$, then $\omega(t)=-\frac{1}{R}$ if $\lambda_{3}(t)>0, \forall t \in[s, T)$ and $\omega(t)=\frac{1}{R}$ if $\lambda_{3}(t)<0, \forall t \in[s, T)$. Thus $\lambda_{3}(t)$ can be determined as:

$$
\begin{aligned}
\lambda_{3}(t)= & \lambda_{3}(T)-\int_{t}^{T} \dot{\lambda}_{3}(s) d s \\
= & \left\{\begin{array}{l}
R\left(1-\frac{\cos \left(\theta(t)-\psi_{T}\right)}{\cos \left(\theta_{T}-\psi_{T}\right)}\right) \text { if } \omega(t)=\frac{1}{R} \\
-R\left(1-\frac{\cos \left(\theta(t)-\psi_{T}\right)}{\cos \left(\theta_{T}-\psi_{T}\right)}\right) \text { if } \omega(t)=-\frac{1}{R}
\end{array}\right. \\
& t \in[s, T) .
\end{aligned}
$$

The following lemma characterizes COTs.

Lemma 3.1: For any terminal state $q_{T}$, a unique COT $q(t)$ and its corresponding input and costate history can be reconstructed. Furthermore, if $\theta_{T} \neq \psi_{T}$, then $q(t)$ is composed of maximumly turning right or left curves, or combination of both at some switching times. If $\theta_{T}=\psi_{T}$, then $q(t)$ is a line that goes through the origin.

A direct consequence of the Lemma 3.1 is that, an optimal trajectory can not contain both a circular arc and a line segment. Furthermore, from the proof of Lemma 3.1, it was shown that if a COT contains at least one switching point, then the angle of first switching satisfies the following equation:

$$
\theta\left(t_{s}\right)=-\left(\theta_{T}-\psi_{T}\right)+\psi_{T}
$$

To ease analysis of optimal trajectories, it is useful to rotate the exit point $q_{T}$ clockwise by $\psi_{T}$ to $\tilde{q}_{T}$, so that after rotation $\tilde{x}_{T}=r, \tilde{y}_{T}=0, \tilde{\theta}_{T}=\theta_{T}-\psi_{T}$. In this case $\tilde{\psi}_{T}=0$ and $\tilde{\theta}_{T} \in\left(-\frac{\pi}{2}, \frac{\pi}{2}\right)$. Since the costate equation does not change under rotation (function of relative angle differences), the corresponding COT is simply rotated as well.

For a number of terminal conditions $q_{T}$, the corresponding COTs are shown in Figure 2. The state in $x-y$ plane $[x(t), y(t)]$, costate $\lambda_{3}(t)$ and angle $\theta(t)$ is plotted from left to right. From Figure 2, it can bee seen that the angle $\theta(t)$ bounces between $\theta_{T}$ and $-\theta_{T}$. Going back in time and starting at $\theta_{T}$, the COT switches every time $\theta(t)$ reaches $-\theta_{T}$ or $\theta_{T}$ again.

After rotation to $\left[r, 0, \theta_{T}\right]^{T}$, using the analysis outlined in the proof of Lemma 3.1 (see [3]), the i-th switching time $t_{s}^{i}$ and switching angle $\theta\left(t_{s}^{i}\right)$ starting from time $T$ can be
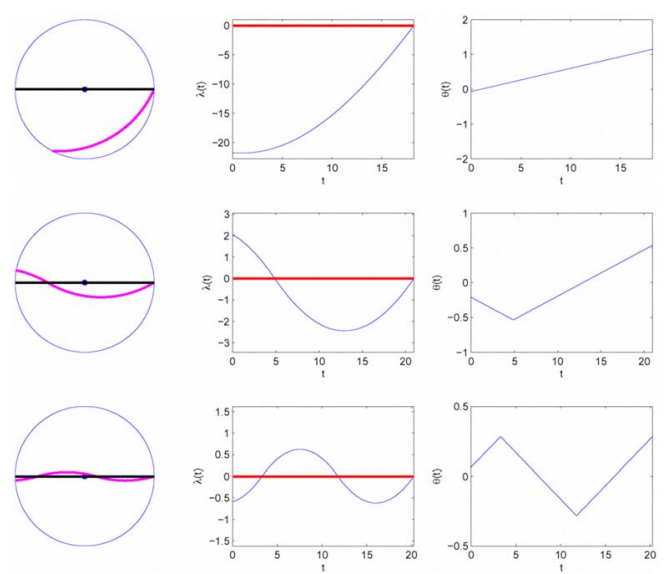

Fig. 2. A number of $q(t)$ is plotted for different terminal conditions $q_{T}$ resulting in different number of switches. The left plot is the state in $x-y$ plane, the middle plot is the costate $\lambda_{3}$ and the right plot is the angle $\theta(t)$.

characterized as:

$$
\begin{aligned}
T-t_{s}^{i} & =2 R\left|\theta_{T}\right| i \\
\theta\left(t_{s}^{i}\right) & =-\theta_{T}, i \text { odd } \\
\theta\left(t_{s}^{i}\right) & =\theta_{T}, i \text { even }
\end{aligned}
$$

We denote $T$ as the 0 -th switching time. Using equations (19), the following lemma can be proven. This lemma is useful to obtain a simple and geometric law to determine optimal switching points.

Lemma 3.2: For any initial condition $q(0)$, all switching points of the optimal trajectory lie on the line passing through the origin and the exit point.

Lemma 3.2 is then used to establish an important characterization of optimal trajectories as described in the next theorem.

Theorem 3.3: For any initial condition $q(0)$, the optimal trajectory of the UAV does not switch more than once.

Since the optimal trajectory can only switch at most once, the number of COT that can be optimal is drastically reduced. It is then possible to construct optimal curves for any initial condition in the feasible set $\Lambda$. Similar to many other Dubins car path planning approaches (see [4], [14], [10], [2] for example), we can define 2 motion primitives $\{L, R\}$, where $L$ and $R$ motion primitives turn the car maximumly to the left and right, respectively. For this problem, there is only one case where a straight line is a COT (initial condition $q(0)=[-r, 0,0]^{T}$ ). However, in this case, there are 2 other COTs that are both shorter in length and involve oneswitching. Therefore unlike the Dubins vehicle shortest-path problem ([10]), there is no straight line motion primitive. Furthermore, since the optimal trajectory only switch once, there are only 4 possible sequences of the $\{L, R\}$ motion 
primitives, namely

$$
\{L, R, L R, R L\} \text {, }
$$

where $L R$ stands for turning left then right and $R L$ for turning right then left. There are two ways to determine the optimal switching point. The first one is geometric. As Lemma 3.2 states, the switching point must be on the same line as the origin and point of exit (this exit point can be determined by projecting the state either turning left or right until exiting the convoy circle). The second way is to check for the heading $\theta(t)$, which is required to satisfy equation (18). Since there is only 4 possibilities for the motion sequences in an optimal trajectory, it is easy to determine the global optimal path for any initial condition. A set of optimal paths for initial conditions with heading $\theta(0)=\frac{\pi}{2}$ are shown in Figure 3 . Figure 3 also shows the optimal switching surface on which switchings are optimal.

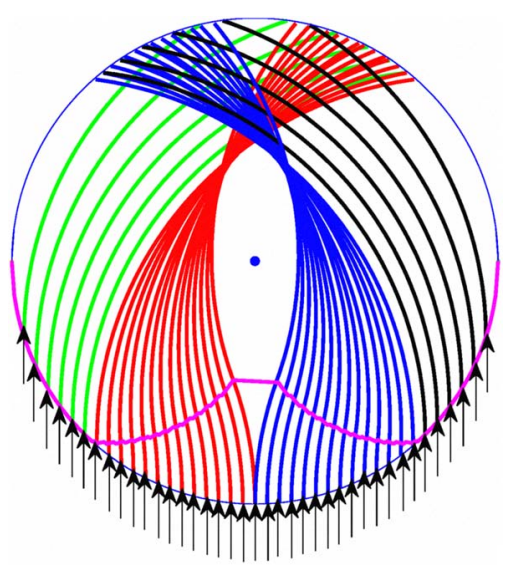

Fig. 3. A number of optimal state trajectories with initial heading $\frac{\pi}{2}$. The optimal motion sequence depends on the initial position. If $x(0) \in\left(-r,-\frac{r^{2}}{R}\right]$, then $R$ is optimal. If $x(0) \in\left(-\frac{r^{2}}{R}, 0\right]$, then $L R$ is optimal. If $x(0) \in\left[0, \frac{r^{2}}{R}\right)$ then $R L$ is optimal. If $x(0) \in\left[\frac{r^{2}}{R}, r\right)$, then $L$ is optimal. The optimal switching points are plotted together to form the optimal switching surface. In this case, $R=15, r=10$.

The above switching surface provides a control law which produces the optimal trajectory for any given initial condition. However, a more important problem is to find the optimal path inside the convoy circle with initial condition free. Hence the problem of finding

$$
q^{*}(0)=\arg \min \Pi_{q(0)} .
$$

The optimal path with this initial condition is denoted as a globally optimal path. $q^{*}(0)$ is referred to as an optimal entry point. It is apparent that any rotation of this point around the origin is also an optimal entry point. This set of optimal entry points, denoted by $\mathbb{Q}^{*}$, can be exactly determined by the following theorem.

Theorem 3.4: The angle of the optimal entry point $q^{*}(0)$ satisfies the equation:

$$
\theta^{*}(0)-\psi_{T}^{*}=-\left(\theta_{T}^{*}-\psi_{T}^{*}\right)
$$

From the proof of Theorem 3.4 (see [3]) we saw that all entry points are rotations of the two points: $q(0)=$ $\left[-r, 0, \arcsin \left(\frac{r}{R}\right)\right]^{T}$ and $q(0)=\left[-r, 0,-\arcsin \left(\frac{r}{R}\right)\right]^{T}$. By rotating these two points around the origin, the optimal entry point set $\mathbb{Q}^{*}$ can be obtained and it is represented in the following form:

$$
\begin{aligned}
\mathbb{Q}^{*}= & \left\{q=\left[-r \cos (\theta),-r \sin (\theta), \pm \arcsin \left(\frac{r}{R}\right)-\theta\right]^{T},\right. \\
& \theta \in[-\pi, \pi]\}
\end{aligned}
$$

An easy way to recognize a globally optimal path is to observe the fact that the entry point of a globally optimal path is always on the same line as the origin and the exit point.

\section{Multi-UAV Convoy Protection}

Due to kinematic constraint of the UAVs $(r<R)$, it is impossible for one UAV to provide complete convoy protection for a group of UGVs. In this situation, multi-UAV coordination is required in order to successful carry out convoy protection. The last section laid out the ground-work to achieve optimal convoy protection by a group of UAVs. Theorem 3.4 characterized a set of optimal initial conditions that produces a set of globally time-optimal trajectory. It can be shown that these optimal trajectories not only specifies a path inside the convoy circle, but also a path for a single UAV to come back to the convoy circle without changing direction. As shown in Figure 4 , the path constitutes a circle of radius $R$ and part of the path is the optimal trajectory inside the convoy circle. There are many similar optimal paths, and they are referred to as optimal convoy protection paths. These paths maximize the ratio of time inside the convoy circle over outside of the convoy circle, since it is the quickest path to come back to the circle, always reenter optimally and repeat as a limit-cycle.

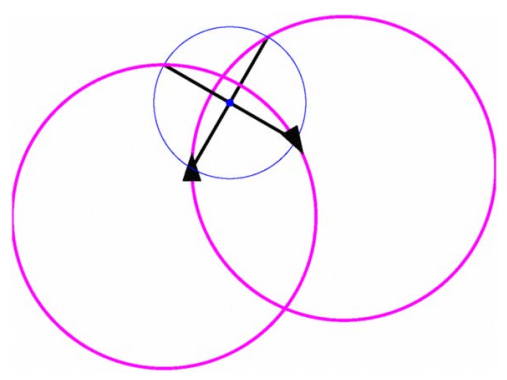

Fig. 4. Two optimal convoy protection paths are shown. They maximize the time spent inside the convoy circle over the time outside of the convoy circle. The triangles indicates position of the UAVs on the paths. The smaller circle is the convoy circle, and the larger circles are optimal convoy protection paths.

To ensure that all the UAVs maximize their time providing convoy protection, their paths should be set to optimal convoy protection paths such as the ones shown in Figure 4. In order to achieve successful convoy protection, it is required that the 
UGVs are visible to at least one UAV at all time. Thus, we can establish a lower bound on the number of UAV required to provide successful convoy protection based on the optimal path length.

Corollary 4.1: Given the convoy circle of radius $r$ for the UGVs and maximum turning radius $R$ for the UAVs, the minimum number of UAV needed to provide convoy protection for all time is:

$$
N=\left\lceil\frac{\pi}{\arcsin \left(\frac{r}{R}\right)}\right\rceil,
$$

where $\lceil\cdot\rceil$ denotes the ceiling function.

Assume that there is $N$ UAVs and they can start at an optimal initial condition $q^{*}(0) \in \mathbb{Q}^{*}$, the UAVs need to space themselves evenly in terms of the time entering the convoy circle. This can be achieved by slowing down and speeding up with respect to the other UAVs so that the $i$-th UAV enters the convoy circle at time $\frac{2 \pi R}{N} i$. This strategy is possible since the optimal paths derived for this problem remain the same for UAVs of any speed (instead of unit speed).

\section{CONCLUding REMARKS}

In this paper we have obtained optimal paths for a single UAV so that it provides ground convoy protection for maximum time. We also obtained optimal paths for multiple UAVs and the minimum number of UAV needed to provide convoy protection to ground vehicles for all time.

\section{ACKNOWLEDGEMENTS}

This work was sponsored by a grant from Rockwell Collins Advanced Technology Center.

\section{REFERENCES}

[1] C. Belta, A. Bicchi, M. Egerstedt, E. Frazzoli, E. Klavins, and G. J. Pappas. Symbolic planning and control of robot motion: State of the art and grand challenges. IEEE Robotics and Automation Magazine, Vol. 14, No. 1, pp. 61-70, 2007.

[2] H. Chitsaz, S. M. LaValle. Time-optimal paths for a Dubins airplane, IEEE Conference on Decision and Control, pp. 2379-2384, Dec. 2007.

[3] X. C. Ding, A. Rahmani and M. Egerstedt. Optimal Multi-UAV Convoy Protection. Technical report, GRITS Lab, Georgia Institute of Technology, http://www.ece.gatech.edu/ ding/technicalreport.pdf (2008)

[4] L. Dubins. On curves of minimal length with a constraint on average curvature, and with prescribed initial and terminal positions and tangents, American Journal of Mathematics, Vol. 79, pp. 497-516, 1957.

[5] L. E. Dubins. On plane curves with curvature. Pacific J. Math., Vol. 11, No. 2, pp. 471-481, 1961

[6] E. Frazzoli, M. A. Dahleh, and E. Feron. Maneuver-based motion planning for nonlinear systems with symmetries. IEEE Trans. on Robotics, Vol. 21, No. 6, pp 1077-1091, 2005.

[7] B. Grocholsky, J. Keller, V. Kumar and G. Pappas. Cooperative Air and Ground Surveillance: A scalable approach to the detection and localization of targets by a network of UAVs and UGVs. IEEE Robotic and Automation Magazine, Vol. 13, No. 3, pp. 16-26, 2006.

[8] K. Hauser, T. Bretl, K. Harada, and J. C. Latombe. Using motion primitives in probabilistic sample-based planning for humanoid robots. In Workshop on the Algorithmic Foundations of Robotics (WAFR), 2006.

[9] D. E. Kirk. Optimal Control Theory, An Introduction, Dover Publications, 2004.

[10] S.M. Lavalle. Planning Algorithms, Cambridge University Press, NY, 2006.
[11] J. Reeds and L. Shepp. Optimal paths for a car that goes both forwards and backwards. Pacific J. Math., Vol. 145, No. 2, pp. 367-393, 1990.

[12] K. Savla, E. Frazzoli, and F. Bullo. Traveling Salesperson Problems for the Dubins vehicle. IEEE Trans. on Automatic Control, Vol. 53, No. 6, pp.1378-1391, 2008.

[13] P. Souèeres and J. Laumond. Shortest paths synthesis for a car-like robot. IEEE Transactions on Automatic Control, Vol. 41, No. 5, pp. 672-688, 1996.

[14] P. Souèeres and J. Boissonnat. Optimal trajectories for nonholonomic mobile robots. Robot Motion Planning and Control, pp. 93-170. Springer, 1998.

[15] S. C. Spry, A. R. Girard and J. K. Hedrick. Convoy protection using multiple Unmanned Air Vehicles: Organization and Coordination. American Control Conference, Portland, Oregon, Jun. 2005.

[16] G. Walsh, A. Sarti, and S. Shankar Sastry. Algorithms for steering on the group of rotations. Technical Report UCB/ERL M93/44, EECS Department, University of California, Berkeley, 1993. 